\title{
Lattice Boltzmann Simulation of High-Frequency Flows: Electromechanical Resonators in Gaseous Media
}

\author{
Carlos Colosqui ${ }^{*}$, Devrez M. Karabacak ${ }^{2}$, Kamil L. Ekinci $^{1}$ And Victor Yakhot ${ }^{1}$ \\ ${ }^{1}$ Department of Mechanical Engineering, Boston University, Boston, MA 02215, USA \\ ${ }^{2}$ IMEC Holst Centre, Eindhoven, 5605 KN, The Netherlands
}

April 2009

\begin{abstract}
In this work, we employ a kinetic theory based approach to predict the hydrodynamic forces on electromechanical resonators operating in gaseous media. Using the Boltzmann-BGK equation, we investigate the influence of the resonator geometry on the fluid resistance in the entire range of nondimensional frequency variation $0 \leq \tau \omega \leq \infty$; here the fluid relaxation time $\tau=\mu / p$ is determined by the gas viscosity $\mu$ and pressure $p$ at thermodynamic equilibrium, and $\omega$ is the (angular) oscillation frequency. Our results support the experimentally observed transition from viscous to viscoelastic flow in simple gases at $\tau \omega \approx 1$. They are also in remarkable agreement with the measured geometric effects in resonators in a broad linear dimension, frequency, and pressure range.
\end{abstract}

\section{Introduction}

Electromechanical resonators with linear dimensions in the nanometer to micrometer scales are being developed for technological applications and fundamental research. One of the most important attributes of these nano/microelectromechanical systems (N/MEMS) resonators is that they have very small intrinsic dissipation of energy, quantified by their high quality factors $Q_{o}=\mathcal{O}\left(10^{2}-10^{4}\right)$. N/MEMS resonators are thus ultrasensitive to external perturbations enabling important technologies such as atomic force microscopy (AFM) [2] and bio-chemical sensing [10].

Some of the most promising applications of N/MEMS, however, require their immersion in fluid media (e.g., air mixtures or biological fluids), where fluid-device interaction can significantly degrade the overall sensitivity [1, 21, 20]. Numerous efforts are currently underway to overcome this difficulty and develop

\footnotetext{
${ }^{*}$ Present address: Department of Chemical Engineering, Princeton University, Princeton, NJ 08544, USA
} 
future N/MEMS for promising nanotechnological and biomedical applications. Unquestionably, the flows generated by N/MEMS demand a novel understanding of fluid mechanics at increasingly smaller time and length scales. Conversely, experimental characterization and numerical analysis of fluid-immersed N/MEMS resonators provide an invaluable opportunity to advance knowledge in new areas of fluid mechanics such as high-frequency nanofluidics. Recent work on high-frequency oscillating flows [15, 26, 8] reports a viscoelastic transition in simple gases at sufficiently large values of the nondimensional frequency $\tau \omega$. Here, $\tau=\mu / p$ is the relaxation time in terms of the pressure $p$ and viscosity $\mu$ of the gas at equilibrium; $\omega$ is the oscillation frequency. Such remarkable phenomenon is beyond the reach of classical (Newtonian) fluid mechanics, which is only valid for $\tau \omega \ll 1$. The viscoelastic transition is accompanied by a substantial attenuation of the energy dissipated by the fluid and a subsequent improvement in the performance of the fluid-immersed device.

In the present study, we solve the Boltzmann-BGK equation of kinetic theory via appropriate numerical procedures [8, 23] in order to predict fluidic effects (e.g., damping force and energy dissipation) for specific geometries. The validity of the kinetic methods is not constrained to Newtonian flow; thus, our models yield excellent agreement with experimental measurements on different resonators over a wide range of frequency and pressure variation.

\section{Electromechanical resonators}

Illustrated in figures 1(a)-1(b) are the first class of studied resonators in the form of cantilever and doublyclamped beams $\left(L_{z} \gg L_{x} \sim L_{y}\right)$. Harmonic motion in the beams can be induced through the application of periodic electrostatic, photothermal, or inertial forces. The beams are driven around their fundamental and first harmonic out-of-plane flexural resonances while optical techniques are used to determine the resonant response [16]. The doubly-clamped beams are suspended above a stationary substrate at a mean height $\Delta \simeq 400 \mathrm{~nm}$; thus, the presence of the substrate has no significant effect on the fluidic damping (e.g. via squeeze-film damping) [15]. We also study a macroscopic quartz crystal resonator [11]. The studied resonator [see figure 1(c)] is in the form of a thin crystal disk $\left(L_{x}=L_{z} \gg L_{y}\right)$ connected to electrodes so that its resonances in thickness-shear modes can be electrically excited and detected by piezoelectric effects. In all the measurements, the resonance amplitudes of the beams and crystal resonators are kept extremely small.

Specific dimensions and (vacuum) characteristics, such as resonance frequency $\omega_{o}$, quality factor $Q_{o}$, and surface to modal mass ratio $S / m_{o}$, of four studied resonators are listed in table 2. The size of the devices vary from sub-micron to millimeters while their resonance frequencies are in the range of kilohertz to megahertz. Experimental analysis of the four resonators in table 2 is performed with the devices operating in purified nitrogen at room temperature $T \simeq 300 \mathrm{~K}$; the pressure is gradually varied from low-vacuum to atmospheric pressure, $0.1 \leq p \leq 1000$ Torr. As the pressure is varied and given that $\tau=\mu / p$, the resulting flows cover a wide range of dimensionless frequency variation $0.001 \leq \tau \omega \leq 10$ [15]. 


\begin{tabular}{|c|c|c|c|c|c|}
\hline $\begin{array}{l}\text { Class } \\
\text { ( : first harmonic) }\end{array}$ & $\begin{array}{l}\text { Dimensions } \\
\qquad(\mu \mathrm{m})\end{array}$ & $\begin{array}{l}\omega_{o} / 2 \pi \\
(\mathrm{MHz})\end{array}$ & $Q_{o}$ & $\begin{array}{c}S / m_{o} \\
\left(\mathrm{~m}^{2} / \mathrm{kg}\right)\end{array}$ & $A R$ \\
\hline Cantilever Beam & $L_{x}=2.0 \quad L_{y}=53 \quad L_{z}=460$ & 0.078 & 8320 & 688 & 26 \\
\hline Cantilever Beam & $L_{x}=3.6 \quad L_{y}=36 \quad L_{z}=125$ & 1.97 & 3520 & 405 & 10 \\
\hline Doubly-Clamped Beam & $L_{x}=0.2 \quad L_{y}=0.23 L_{z}=9.6$ & 24.2 & 415 & 8380 & 1 \\
\hline Quartz Crystal Disk & $L_{x}=L_{z}=d \simeq 10000 L_{y} \simeq 160$ & 32.7 & 40755 & 6.29 & 0 \\
\hline
\end{tabular}

Table 1: Electromechanical Resonators



Figure 1: (a) Cantilever Beam (b) Doubly-Clamped Beam (c) Quartz Crystal 
Resonators immersed in a fluid. The dynamics of an electromechanical resonator immersed in a fluid can be studied by means of a one-dimensional harmonic oscillator approximation [7]

$$
m_{o}\left(x_{t t}+\gamma_{o} x_{t}+\omega_{o}^{2} x\right)=F(t)+F_{f}(t)
$$

where $m_{o}$ is the effective mass corresponding to the vibrational mode [15, 7], $\gamma_{o}$ is the (structural) damping coefficient and $\omega_{o}$ is the resonance frequency of the device in vacuum. The load on the oscillator is produced by the driving force $F(t)=\operatorname{Re}\left\{F(\omega) e^{-i \omega t}\right\}$ along with a fluid resistance $F_{f}(t)=-m_{o}\left(\gamma_{f} x_{t}+\beta_{f} x_{t t}\right)$ which has both dissipative and inertial components [17]. The oscillation amplitude has the general form $x(t)=\operatorname{Re}\left\{x(\omega) e^{-i(\omega t+\phi)}\right\}$ and from equation (1) the frequency response is

$$
x(\omega)=\frac{F_{0}}{m_{o}} \frac{1}{\left[\omega_{o}^{2}-\omega^{2}\left(1+\beta_{f}\right)-i \omega\left(\gamma_{o}+\gamma_{f}\right)\right]},
$$

with $F_{0} \equiv F(\omega) e^{i \phi}$ being the effective force amplitude. Equation (2) includes fluidic effects through the fluidic inertia, or fluid-added mass, $\beta_{f}$ and fluidic damping $\gamma_{f}$. Experimental values of the added mass and fluidic dissipation are respectively obtained from the frequency shift and the broadening of the Lorentzian frequency response (2) [15]. In this study, the fluid-added mass is very small $\beta_{f} \ll 1$ and so is the measured shift in the resonance frequency: $\Delta \omega_{o} / \omega_{o} \approx \beta_{f} / 2$. The device quality factor in the fluid thus becomes $Q \approx \omega_{o} /\left(\gamma_{o}+\gamma_{f}\right)$.

\section{$3 \quad$ Hydrodynamics of high-frequency flows}

Similar to previous work [1, 21, 20], the present analysis is valid when gradients along the spanwise direction of the oscillating body are negligible so that the flow is considered two-dimensional. This assumption holds for a slender beam $\left(L_{z} \gg L_{x} \sim L_{y}\right)$ with uniform rectangular cross-section, or a thin disk $\left(L_{x}=L_{z}=d \gg L_{y}\right)$, where the aspect ratio $A R=L_{y} / L_{x}$ (see table 2) becomes the geometric parameter characterizing the dominant chordwise flow. Other dynamically relevant flow parameters are the Mach number $M=U_{o} / c_{s}$ and the Reynolds number $R e=\rho U_{o} L_{y} / \mu$ determined by the fluid velocity amplitude $U_{o}=|\omega x(\omega)|$, the speed of sound $c_{s} \simeq 298 \mathrm{~m} \mathrm{~s}^{-1}$, and molecular viscosity $\mu \simeq 1.78 \times 10^{-5} \mathrm{~kg} \mathrm{~m}^{-1} \mathrm{~s}^{-1}$ of nitrogen at room temperature. Given the tiny oscillation amplitude of the resonators, the resulting Mach and Reynolds number are extremely small, $M \sim R e<0.001$ in all operation conditions. Consequently, the generated flow can be assumed laminar, nearly incompressible, and isothermal.

The common approach hitherto encountered in the literature [see 1, 21, 20] for the theoretical and/or numerical determination of fluidic effects on resonators is based on the Navier-Stokes equations for Newtonian

fluid flow. However, a fundamental assumption for the applicability of classical Navier-Stokes equations is that hydrodynamic scales are much larger than their kinetic counterparts, i.e. $\tau \omega \ll 1$. Previous work 15, 26, 8, 11] on high-frequency oscillating flows has established that the non-dimensional frequency $\tau \omega$ 
determines qualitatively different behavior: purely viscous (Newtonian) for $\tau \omega=0$, viscoelastic (transitional) for $0<\tau \omega<\infty$, and purely elastic (free-molecular) for $\tau \omega \rightarrow \infty$. While viscous flows are accurately described by Newtonian hydrodynamic equations, an extended hydrodynamic description accounting for kinetic (nonequilibrium) phenomena is required at sufficiently large $\tau \omega$ where non-Newtonian behavior is observed [see [26, $[$ 8].

\subsection{Newtonian hydrodynamics}

In the Newtonian regime $\tau \omega \ll 1$, the flow around a body oscillating with very small amplitude $|x(\omega)| \ll L_{x}$ is governed by the linearized Navier-Stokes (NS) equations for incompressible flow [17]:

$$
\boldsymbol{\nabla} \cdot \mathbf{u}=0, \quad \frac{\partial \mathbf{u}}{\partial t}=\nu \nabla^{2} \mathbf{u}-\frac{1}{\rho} \nabla p .
$$

After adoption of the standard no-slip boundary conditions, these equations provide well-known analytical solutions for simple geometries [17, 24]. Through these solutions, one can determine fluidic forces over a body as

$$
F_{f}(t)=\rho B \omega^{2} \operatorname{Re}\left\{\Gamma(\omega) x(\omega) e^{-i(\omega t+\phi)}\right\},
$$

where $\rho$ is the fluid density and $B$ is the body volume; $\Gamma=m_{o}\left(\beta_{f}+i \gamma_{f} / \omega\right) /(\rho B)$ is the so-called hydrodynamic function. One of the simplest solutions of the unsteady NS equations is obtained for an infinite plate oscillating in a fluid $\left(L_{x}=L_{z}=\infty, L_{y}=0\right)$, known as the Stokes' second problem [26, 17]. For a slender body having a thin cross section with small but finite width $(0<A R \ll 1)$ the Newtonian hydrodynamic function can be approximated by the solution of the Stokes' second problem:

$$
\Gamma_{(A R \ll 1)}(\omega)=(1+i) \frac{S}{B} \sqrt{\frac{\nu}{2 \omega}} .
$$

Here, $S$ is the surface area in contact with the fluid and $\nu=\mu / \rho$. When the cross section is not small $(A R \gtrsim 1)$, the common approach has been to study the flow generated by simpler geometries such as a cylinder with a radius equal to half the nominal length scale, $r=L_{y} / 2$. For the case of an infinite cylinder with its axis normal to the $x$-direction, the hydrodynamic function is $[1,21,20]$

$$
\Gamma_{c y l}(\omega)=1+\frac{4 i}{\sqrt{i \omega r^{2} / \nu}} \frac{\mathrm{K}_{1}\left(-i \sqrt{i \omega r^{2} / \nu}\right)}{\mathrm{K}_{0}\left(-i \sqrt{i \omega r^{2} / \nu}\right)},
$$

where $K_{0}$ and $K_{1}$ are Bessel's functions of the third kind. The asymptotic behavior of the Newtonian hydrodynamic function of a cylinder (5) and that of a rectangular beam are similar in both limits $\omega r^{2} / \nu \rightarrow 0$ and $\omega r^{2} / \nu \rightarrow \infty$ 24]. For this reason, and only within the Newtonian regime $(\tau \omega \ll 1)$, the solution for an oscillating cylinder has been employed with some degree of accuracy in estimating fluidic effects on rectangular beams with cross-sections of moderate to large aspect ratio [1, 21, 20]. For very large aspect ratios $\left(L_{x} \rightarrow 0\right)$, 21] formulated an empirical correction to equation (5) such that the hydrodynamic function becomes $\Gamma_{(A R \gg 1)}=\Gamma_{c y l} \Omega\left(\omega L_{y}^{2} / \nu\right)$. Nevertheless, the correction of [21] remains essentially unity $|\Omega-1|=\mathcal{O}\left(10^{-1}\right)$ within all regimes studied in this work. 


\subsection{Beyond Newtonian hydrodynamics}

When flow time scales $\mathcal{T}=1 / \omega$ are of the same order as the relaxation time $\tau$, kinetic effects become significant and Newtonian hydrodynamic equations (3) breakdown. The primary issue encountered beyond Newtonian regimes $(\tau \omega \gtrsim 1)$ is the lack of a robust hydrodynamic equation governing the flow. To obtain a hydrodynamic description valid for arbitrary nondimensional frequencies $(0 \leq \tau \omega \equiv \tau / \mathcal{T} \leq \infty)$, one must resort to kinetic theory representations of the flow. Unfortunately, the problem of deriving (closed-form) hydrodynamic equations via kinetic theory, albeit largely studied [see 4, 13, 5, 6], remains essentially open for arbitrary flow regimes.

Another critical point arising when kinetic effects are no longer negligible is that of proper boundary conditions for the hydrodynamic equations at the solid-fluid interface. Hydrodynamic boundary conditions are determined by a rather complex fluid-solid interaction in the vicinity of a solid surface. For flows at finite Knudsen number, the slip boundary condition has been extensively adopted [4, 18, 25]. According to Maxwell's picture of slip of a gas over a solid surface, a finite mean-free-path $\lambda \sim \tau c_{s}$ leads to an effective slip velocity

$$
\left[\mathbf{u}\left(\mathbf{x}_{w}, t\right)-\mathbf{U}_{w}\right] \cdot \mathbf{t}=\frac{2-\sigma_{v}}{\sigma_{v}} \lambda[\boldsymbol{\nabla}(\mathbf{u} \cdot \mathbf{t}) \cdot \mathbf{n}+\boldsymbol{\nabla}(\mathbf{u} \cdot \mathbf{n}) \cdot \mathbf{t}]
$$

to be employed as boundary condition at the coarse-grained (hydrodynamic) level. Here $\mathbf{t}$ and $\mathbf{n}$ are the unit tangent and normal vectors to a wall located at $\mathbf{x}_{w}$ and moving with velocity $\mathbf{U}_{w} ; \mathbf{u}$ is the fluid velocity. Meanwhile, $\sigma_{v}$ is the tangential momentum accommodation coefficient of the solid surface. A unit accommodation coefficient $\left(\sigma_{v}=1\right)$ represents a situation where all fluid particles are diffusively scattered after collision with the wall; the opposite limit $\left(\sigma_{v}=0\right)$ corresponds to the case where all such collisions are specular. First-order Maxwell slip models (6) are accurate for steady flow at small to moderate Knudsen numbers $(K n<1)[25$, 19] while high-order versions have been proposed for unsteady shear flow [19, 14]. The effective slip in oscillating shear flows has recently been investigated via kinetic methods such as lattice Boltzmann-BGK (LBGK) and direct simulation Monte Carlo (DSMC). Expected functional shapes have been obtained for the slip as a function of the Knudsen number $K n=\lambda / \mathcal{L}$ in steady shear flows $\left(\mathcal{L} \sim\left|\nabla u_{t}\right| /\left|u_{t}\right|\right)$ or the nondimensional frequency $\tau \omega=\tau / \mathcal{T}$ in oscillating shear flows [19, 14, 9$]$.

\section{Kinetic model of hydrodynamics}

At room temperature and under ordinary pressure conditions (ranging from low-vacuum to atmospheric pressure), simple gases are composed of a large number of electrically neutral molecules, each with an effective diameter that is negligible compared to the average intermolecular distance. Under such conditions the Boltzmann-BGK equation (BE-BGK) is an accurate kinetic model of the flow [4, 6]. For monatomic gases in the absence of external force fields $\mathbf{F}=0$, the BE-BGK for the evolution of the Boltzmann distribution 
$f(\mathbf{x}, \mathbf{v}, t)$ in phase space $(\mathbf{x}, \mathbf{v})$ reads:

$$
\frac{\partial f}{\partial t}+\mathbf{v} \cdot \nabla f=-\frac{f-f^{e q}}{\tau} .
$$

Without loss of generality, we define $\theta=k_{B} T / m_{\text {gas }}=c_{s}^{2}$ and adopt a molecular mass $m_{\text {gas }}=1$; the equilibrium distribution can then be expressed as

$$
f^{e q}(\mathbf{x}, \mathbf{v}, t)=\frac{\rho}{(2 \pi \theta)^{\frac{D}{2}}} \exp \left[-\frac{(\mathbf{v}-\mathbf{u})^{2}}{2 \theta}\right]
$$

where $D$ is the velocity space dimension $\left(\mathbf{v}=v_{k} \mathbf{e}_{k} ; k=1, D\right)$. Hydrodynamic quantities, like mass density $\rho$, fluid velocity $\mathbf{u}$, and energy are obtained as moments of the distribution function:

$$
\begin{aligned}
\int f(\mathbf{x}, \mathbf{v}, t) d \mathbf{v} & =\rho(\mathbf{x}, t) \\
\int f(\mathbf{x}, \mathbf{v}, t) \mathbf{v} d \mathbf{v} & =\rho \mathbf{u}(\mathbf{x}, t) \\
\int f(\mathbf{x}, \mathbf{v}, t) \mathbf{v}^{2} d \mathbf{v} & =\rho D \theta(\mathbf{x}, t)+\rho \mathbf{u}^{2}(\mathbf{x}, t) .
\end{aligned}
$$

Kinetic boundary conditions. For bounded flows, particular solutions of equation (7) will require proper boundary conditions. Within the framework of classical kinetic theory we consider the gas as bounded by a perfectly elastic and isothermal surface $\left(\theta_{w}=\theta\right)$ located at $\mathbf{x}_{w}$ while moving with velocity $\mathbf{U}_{w}$. Under this depiction, particles impinging on a solid surface with velocity $\mathbf{v}^{\prime}$ acquire a post-collision velocity $\mathbf{v}$ defined by the scattering kernel $B\left(\mathbf{v}^{\prime} \rightarrow \mathbf{v}\right)$. General boundary conditions will then read $[4]$ :

$$
\left|\left(\mathbf{v}-\mathbf{U}_{w}\right) \cdot \mathbf{n}\right| f\left(\mathbf{x}_{w}, \mathbf{v}, t\right)=\int_{\left(\mathbf{v}^{\prime}-\mathbf{U}_{w}\right) \cdot \mathbf{n}<0}\left|\left(\mathbf{v}^{\prime}-\mathbf{U}_{w}\right) \cdot \mathbf{n}\right| B\left(\mathbf{v}^{\prime} \rightarrow \mathbf{v}\right) f\left(\mathbf{x}_{w}, \mathbf{v}^{\prime}, t\right) d \mathbf{v}^{\prime} ;
$$

for $\left(\mathbf{v}-\mathbf{U}_{w}\right) \cdot \mathbf{n}>0$. In this work we implement and assess two different kinetic boundary conditions at the fluid-solid interface; diffuse scattering (DS)

$$
f\left(\mathbf{x}_{w}, \mathbf{v}, t\right)=\frac{\rho}{(2 \pi \theta)^{\frac{D}{2}}} \exp \left[-\frac{\left(\mathbf{v}-\mathbf{U}_{w}\right)^{2}}{2 \theta}\right] ; \quad\left(\mathbf{v}-\mathbf{U}_{w}\right) \cdot \mathbf{n}>0
$$

and bounce-back (BB)

$$
f\left(\mathbf{x}_{w}, \mathbf{v}, t\right)=f\left(\mathbf{x}_{w},-\mathbf{v}+2 \mathbf{U}_{\mathbf{w}}, t\right) ; \quad\left(\mathbf{v}-\mathbf{U}_{w}\right) \cdot \mathbf{n}>0
$$

where impinging particles $\left[\left(\mathbf{v}-\mathbf{U}_{w}\right) \cdot \mathbf{n}<0\right]$ are reflected back with the same relative speed and angle of incidence. While a diffuse-scattering (DS) kernel yields hydrodynamic slip in agreement with the Maxwell slip model (6) for a fully accommodating surface $\left(\sigma_{v}=1\right)$, the bounce-back model renders no-slip at the wall for all flow regimes (i.e. the surface has negative accommodation coefficient $\sigma_{v}=-2$ ). The validity range of each model for the solid-fluid interaction will be assessed when comparing LBGK simulation using both DS and $\mathrm{BB}$ schemes against experimental results. 


\subsection{Free-molecule hydrodynamics}

The nondimensional frequency $\tau \omega=2 \pi \lambda / \mathcal{L}_{F M}$ is proportional to the ratio of the (equilibrium) mean free path $\lambda=\tau c_{s}$ to the average distance $\mathcal{L}_{F M}=c_{s} 2 \pi / \omega$ traveled by a particle during one oscillation period. Hence, in the limit $\tau \omega \rightarrow \infty$, the kinetic dynamics becomes practically collisionless and free-molecular flow approximations are applicable. Let us now analyze a $L_{x} \times L_{y}$ rectangular section in free molecular flow moving in the $x$-direction at velocity $\mathbf{u}=u \mathbf{i}$ such that $M=U_{w} / \sqrt{\theta} \ll 1$. In the case of diffusive wall scattering (11) the net $x$-force per unit length is [3]

$$
F_{f}^{D S}(t)=-\left(\sqrt{\frac{8}{\pi}} L_{y}+\sqrt{\frac{2}{\pi}} L_{x}\right) \rho \sqrt{\theta} u .
$$

For the bounce-back model (12), which renders no hydrodynamic slip, one has $F_{f}^{B B}=2 F_{f}^{D S}$. Similar expressions can be obtained for other models of the gas-surface interaction, e.g. $F_{f}^{S P}(t)=-\left(\sqrt{\frac{32}{\pi}} L_{y}\right) \rho \sqrt{\theta} u$ for specular reflection models rendering no shear stress. Clearly, in free molecular flow $(\tau \omega \rightarrow \infty)$, there is no fluidic inertia $\left(\beta_{f} \rightarrow 0\right)$ and hydrodynamic forces only have a dissipative component $\left(F_{f}=f_{d} u\right)$.

\section{Lattice Boltzmann BGK simulation}

The method in this work falls in the class of high-order LBGK models originally formulated by [23, 22] and investigated by [8] for isothermal unidirectional flow in non-Newtonian regimes. Discretization of velocity space in a finite number of lattice velocities $\left\{\mathbf{v}_{i} ; i=1, Q\right\}$ allows one to reduce the problem of solving equation (7) to that of solving a set of lattice Boltzmann-BGK (LBGK) equations

$$
\begin{gathered}
\frac{\partial f_{i}}{\partial t}+\mathbf{v}_{i} \cdot \nabla f_{i}=-\frac{f_{i}-f_{i}^{e q}}{\tau} \\
f_{i}^{e q}=w_{i} \rho\left[1+\frac{1}{\theta}\left(\mathbf{v}_{i} \cdot \mathbf{u}\right)+\frac{1}{2 \theta^{2}}\left(\mathbf{v}_{i} \cdot \mathbf{u}\right)^{2}-\frac{1}{2 \theta} \mathbf{u}^{2}\right] \quad i=1, Q
\end{gathered}
$$

governing the evolution of each lattice population $f_{i}(\mathbf{x}, t)=w_{i} f\left(\mathbf{x}, \mathbf{v}_{i}, t\right)$ in configuration space. The set of LBGK equations (14, 15) is formally derived by projecting the continuum Boltzmann-BGK equation (7] 8 onto the Hilbert space $\mathrm{H}^{2}$ spanned by the orthonormal basis of Hermite polynomials up to second order. A Gauss-Hermite quadrature formula determines the lattice velocities $\mathbf{v}_{i}$, i.e. integration points, and their associated weights $w_{i}$. A quadrature rule with algebraic degree of precision $d \geq 4$ permits the exact numerical integration of the three leading moments (10) of the continuum distribution $f$ :

$$
\rho(\mathbf{x}, t)=\sum_{i=1}^{Q} f_{i}(\mathbf{x}, t), \quad \rho \mathbf{u}(\mathbf{x}, t)=\sum_{i=1}^{Q} f_{i}(\mathbf{x}, t) \mathbf{v}_{i}, \quad \rho\left(u^{2}+D \theta\right)(\mathbf{x}, t)=\sum_{i=1}^{Q} f_{i}(\mathbf{x}, t) \mathbf{v}_{i}^{2}
$$

The particular lattice employed for the present simulations is the D2Q37 [22] (i.e. velocity space dimension $D=2$, number of lattice velocities $Q=37$ ); the weights and velocity abscissae of the lattice are included in the appendix. After lattice discretization of configuration space $\left(\Delta \mathbf{x}_{i}=\mathbf{v}_{i} \Delta t\right)$ numerical procedures to 
solve the LBGK equations (14) advance in two steps: advection and collision. During the advection step the streaming of lattice populations $\left\{f_{i} ; i=1, Q\right\}$ is performed along the lattice directions:

$$
f_{i}^{a d v}(\mathbf{x}, t)=f_{i}\left(\mathbf{x}-\mathbf{v}_{i} \Delta t, t\right)
$$

At the end of the advection step, mass, momentum, and energy (16) are computed using $f_{i}^{a d v}$ allowing the explicit evaluation of equilibrium distributions (15). Then the BGK ansatz is applied at the collision step:

$$
f_{i}\left(\mathbf{x}+\mathbf{v}_{i}, t+\Delta t\right)=f_{i}^{e q}+\left[1-\frac{\Delta t}{\tau}\right] \widehat{f}_{i}^{n e} .
$$

The projected, or dealiased, nonequilibrium component [27]

$$
\widehat{f}_{i}^{n e}=\frac{w_{i}}{2 \theta^{2}}\left(v_{\alpha i} v_{\beta_{i}}-\theta \delta_{\alpha \beta}\right) \sum_{j=1}^{Q}\left(f_{j}^{a d v}-f_{j}^{e q}\right) v_{\alpha j} v_{\beta_{j}} \quad \alpha, \beta=1, D \quad i=1, Q
$$

ensures that the post-collision distribution $f_{i}$ is strictly expressed by a linear combination of up to secondorder Hermite polynomials, i.e $f_{i} \in \mathrm{H}^{2}$. The regularization procedure 27] specified in equations (19)-(18) eliminates significant numerical errors due to lattice orientation anisotropy and aliasing effects that are reported for the standard LBGK algorithm in strong non-equilibrium conditions [8, 27].

\section{Results}

The quantitative analysis of fluidic damping on devices with different sizes and structural features is performed by employing the nondimensional quantity:

$$
\bar{\gamma}_{f}(\tau \omega, A R)=\frac{\gamma_{f}}{\rho \sqrt{\frac{\theta}{2}} \frac{S}{m_{o}}} .
$$

Here, $S / m_{o}$ is the ratio of wet area to modal mass reported in table 2 After normalization by the fluid mass density $\rho$ and speed of sound $c_{s}=\sqrt{\theta}$, the dimensionless damping $\bar{\gamma}_{f}$ (20) is solely determined by the nondimensional frequency $\tau \omega$ and hydrodynamic shape characterized by the aspect ratio $A R=L_{y} / L_{x}$. Based on reported data in table 2, we adopt $A R \simeq 0$ for the quartz resonator and $A R \simeq 1,10 \& 26$ for the beams. Experimentally measured values of the total damping are presented as open symbols in figure 2 These are determined from measurements of the resonant response (2) as a function of pressure as discussed above. As observed in figure 2, experimental values of the density-normalized damping $\bar{\gamma}_{f}$ (20) exhibit an increasing dispersion in the upper limit of dimensionless frequency variation $\tau \omega \rightarrow \infty$ where $\gamma_{f} \rightarrow 0$. This is due to the fact that the measured fluidic effect becomes smaller and smaller as the pressure is lowered and the measured signal is dominated by the finite error $\sim 5 \%$ present in all the experimental data. This is discussed in more detail in [15].

Lattice Boltzmann-BGK (LBGK) simulation. Simulations are performed with the D2Q37-H2 model described in Sec. 5. It has been demonstrated that high-order LBGK models such as D2Q37-H2 with 
a regularization procedure yield excellent agreement with extended hydrodynamic descriptions derived for isothermal and unidirectional flows in both Newtonian and non-Newtonian regimes [8, 27]. In all simulations, $\tau=\mu / p \equiv \nu / \theta$ is readily determined from the gas properties at thermodynamic equilibrium, while the employed Mach number is very low $M=U_{o} / \sqrt{\theta} \leq 0.01$. Simulation results on different resonators obtained by the D2Q37-H2 model for $\tau \omega=0.001,0.01,0.1,0.5,1$, and 10 are compared against experimental data and available analytical expressions for Newtonian and free-molecular flow in figure 2. The employed LBGK models yield a remarkable agreement with experimental measurements over a wide range of nondimensional frequency $0.001 \leq \tau \omega \leq 10$ for different device geometries and dimensions (table 21). On the other hand, Newtonian fluid approximations such as equation (4) for the quartz disk $(A R \simeq 0)$ and (5) for the beams $(A R \geq 1)$ 1, 21, 20] give acceptable agreement only within the low frequency limit $\tau \omega<0.1$. It is important to remark that all Newtonian predictions largely overestimate the fluidic dissipation in the frequency range $\tau \omega>0.1$. In the high-frequency limit $\tau \omega \gg 1$, only kinetic approaches such as LBGK simulation and the free-molecular flow solution given by equation (13) are in good agreement with experiment.

Fluid-solid interaction and boundary schemes. Owing to the small amplitude of oscillation $\left(|x(\omega)| \ll L_{x}\right)$, the solid boundary can be assumed to remain static. Only the boundary velocity $\mathbf{U}_{\mathbf{w}}=U_{o} \sin (\omega t) \mathbf{i}$ varies in time with velocity amplitude $U_{o}<\omega \Delta x$ such that the displacement amplitude is smaller than the distance between neighboring lattice nodes. Two boundary schemes are employed in LBGK simulation when modeling the moving walls: the diffuse-scattering scheme (DS) and the bounce-back scheme (BB) that were explained in Sec. 4. While the BB scheme (12) renders no-slip as hydrodynamic boundary condition, the DS model (11) produces an effective slip velocity between the body surface and the fluid immediately adjacent to it. Experimental results in figure 2 show that numerical schemes rendering no-slip at the wall overpredict the fluidic dissipation when $\tau \omega \geq 0.1$. On the other hand, the diffuse-scattering scheme which models the resonator surface as fully accommodating $\left(\sigma_{v}=1\right)$ yields a close agreement with experimental data in the entire studied range $0.001 \leq \tau \omega \leq 10$.

Quality factor and fluidic effects. Since fluidic inertia is very small $\left(\beta_{f} \ll 1\right)$ for the studied flow conditions the quality factor of the fluid-immersed device is

$$
Q=Q_{o} \frac{1}{1+\rho \sqrt{\frac{\theta}{2}} \frac{S}{m_{o} \gamma_{o}} \bar{\gamma}_{f}(\tau \omega, A R)} .
$$

Evidently, dissipative effects quantified by the structural dissipation $\gamma_{o}=\omega_{o} / Q_{o}$ and fluidic damping $\gamma_{f}=$ $\rho \sqrt{\frac{\theta}{2}} \frac{S}{m_{o}} \bar{\gamma}_{f}$ determine the device performance. The quality factors reported for the four studied devices are compared against numerical predictions in figure 3. We observe that lattice Boltzmann-BGK simulation (model D2Q37-H2 with DS wall treatment) is in close agreement with experimental data obtained for different cross-sections $(0 \leq A R \leq 26)$ in a wide range of operation conditions $\left(0.1 \leq p \leq 1000\right.$ Torr, $0.078 \leq \omega_{o} \leq$ $24 \mathrm{MHz})$. 



Figure 2: Nondimensional fluid damping: $\bar{\gamma}_{f}(\tau \omega, A R)$ vs. $\tau \omega$. (a) $\omega_{o} / 2 \pi=32.7 \mathrm{MHz}$, $A R=0$. (b) $\omega_{o} / 2 \pi=24.2 \mathrm{MHz}, A R=1$. (c) $\omega_{o} / 2 \pi=1.97 \mathrm{MHz}, A R=10$. (d) $\omega_{o} / 2 \pi=0.078$ $\mathrm{MHz}, A R=26$. Open symbols (red): experimental data. Closed dots (black): LBGK simulation $\tau \omega=0.001,0.01,0.1,0.5,1$, and 10; solid line (black): D2Q37-H2 DS; dotted line (black): D2Q37-H2 BB. Dashed line (blue): Newtonian fluid approximations. Dasheddotted line (green): free molecular flow (DS). 


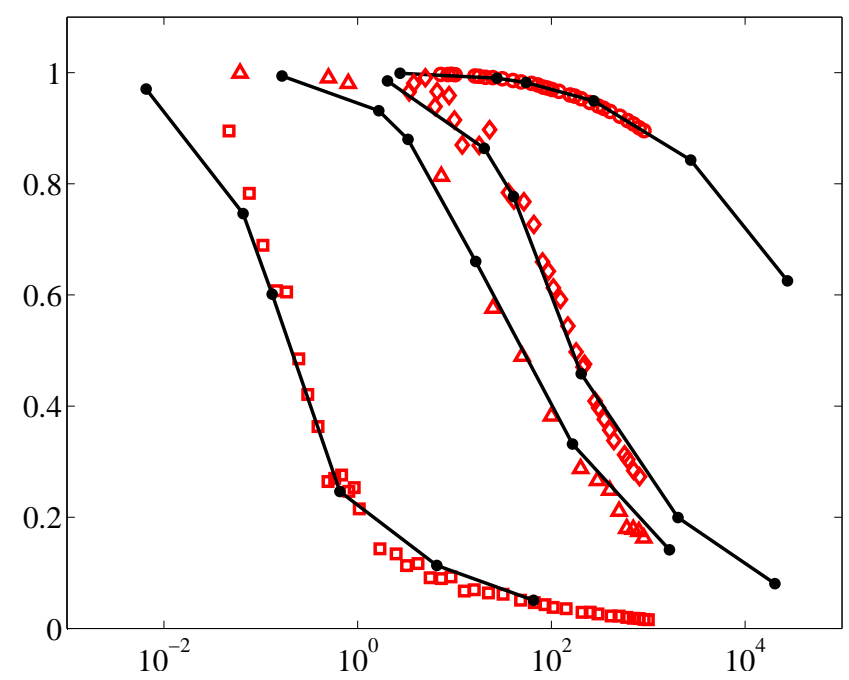

Figure 3: Quality Factor: $\frac{Q}{Q_{o}}=\frac{\gamma_{o}}{\gamma_{o}+\gamma_{f}}$ vs. $p$ [Torr]. Open symbols (red): experimental data; (O) $\omega_{o} / 2 \pi=32.7 \mathrm{MHz}, A R=0 ;(\diamond) \omega_{o} / 2 \pi=24.2 \mathrm{MHz}, A R=1 ;(\triangle) \omega_{o} / 2 \pi=1.97$ $\mathrm{MHz}, A R=10 ;(\square) \omega_{o} / 2 \pi=0.078 \mathrm{MHz}, A R=26$. Closed dots (black): LBGK simulation (D2Q37-H2 DS) $\tau \omega=0.001,0.01,0.1,0.5,1$, and 10.

\section{Conclusions}

Experimental measurements of fluidic effects on diverse electromechanical resonators have been compared against available analytical approaches, i.e. the Newtonian flow and free-molecular flow approximations, as well as against kinetic-based simulations presented in Sec. 5. Clearly, Newtonian and free-molecular flow models fail to describe transitional flow in the region $0.1 \leq \tau \omega \leq 10$. On the other hand, lattice Boltzmann-BGK (LBGK) simulation accounting for specific geometrical features accurately represents the fluidic damping $\gamma_{f}$ in all studied regimes: Newtonian $(\tau \omega \leq 0.1)$, transitional $(0.1 \leq \tau \omega \leq 10)$, and freemolecular flow $(\tau \omega \geq 10)$.

Viscoelastic dynamics in high-frequency flows. The invalidity of Newtonian approaches as $\tau \omega \rightarrow \infty$ is not only due to surface effects, which might be absorbed by proper hydrodynamic boundary conditions, but also due to the qualitatively different fluid dynamics in the bulk [26, 8]. Kinetic-based (LBGK) simulation precisely reproduces the experimentally observed (figure 21) saturation of density-normalized dissipation $\left(\bar{\gamma}_{f} \rightarrow\right.$ const $)$ in the high-frequency limit $\tau \omega \rightarrow \infty$. This remarkable phenomenon involves a gradual transition from viscous to viscoelastic to purely elastic flow of a simple monatomic gas that has been reported by previous theoretical [26, 8] and experimental [15] studies. The viscoelastic response of simple gases in the high-frequency limit is a well-known phenomenon within the realm of transport theory and statistical physics [12]; diffusion processes in nonequilibrium systems can only be established after a finite time $\mathcal{T}_{D} \sim \tau$ of the order of the relaxation time. In the short-time limit $t<\tau$, where diffusion effects are still weak and 
transport coefficients such as shear viscosity become frequency dependent [12], one observes an ensuing decay in the dissipation of fluid momentum and energy.

Near-wall phenomena. Kinetic effects can never be neglected within the so-called Knudsen layer, e.g. at distances from the solid boundary that are smaller than one mean free path. Thus, kinetic parameters, such as the relaxation time and mean free path, are not easily determined in the near-wall region where gas-solid interaction is significant. In consequence, within a mean free path from the wall, Newtonian fluid models for the stress break down and NS equations must actually be applied immediately outside the Knudsen layer. The concept of having effective slip as proper boundary condition for hydrodynamic (coarse-grained) equations must be understood within this context. Our kinetic model of the flow based on the BoltzmannBGK is rather simple and does not accurately represent the Knudsen layer; it relies on a single constant relaxation time $\tau=\mu / p$ and kinetic boundary conditions (10) determined by surface scattering kernels $B\left(\mathbf{v}^{\prime} \rightarrow \mathbf{v}\right)$ for a perfectly elastic and isothermal surface. Nevertheless, the kinetic model in this work accurately predicts hydrodynamic effects such as fluid resistance and mean energy dissipation via adoption of a Maxwell scattering kernel with surface accommodation $\sigma_{v}=1$ (i.e. the DS scheme explained in Sec. 4). The net effect of the studied gas-surface interactions can be assessed by comparing results (figure 2) from DS (slip) and BB (no-slip) schemes in the range $0.001 \leq \tau \omega \leq 10$ for different cross-sections $0 \leq A R \leq 26$. The reduction in fluid damping solely due to effective slip is found in the interval $0-0.4$.

Resonator performance beyond Newtonian regime. The decay of the energy dissipated by the fluid as $\tau \omega \rightarrow \infty$ has beneficial effects on the resonator performance. Under relevant experimental conditions, fluidic damping largely dominates over structural dissipation, $\gamma_{f} \gg \gamma_{o}$, and thus, $Q \approx \omega / \gamma_{f}$. In such conditions the quality factor will actually increase linearly with the operation frequency, $Q \propto \omega$ for $\tau \omega>1$, instead of the square root dependence, $Q \propto \sqrt{\omega}$, observed for Newtonian flow $\tau \omega \ll 1$. Therefore, it is advantageous to operate the resonator at a frequency $\tau \omega>1$ well within the viscoelastic regime. This could be accomplished either by increasing the resonance frequency or by decreasing the effective relaxation time of the fluid through less trivial mechanisms, e.g. through polymer addition or foams for water. Other strategies that can potentially improve the device performance include modifying the cross-sectional shape and surface properties. As observed in figure 2 for the entire range $0.001 \leq \tau \omega \leq 10$, bodies with small aspect ratios $A R=L_{y} / L_{x} \ll 1$ generate less fluidic damping at the same operation frequency and surrounding gas conditions. On the other hand, the employment of superhydrophobic coatings for resonators in water can further increase the effective hydrodynamic slip with a subsequent reduction of the resistance forces.

Lattice Boltzmann-BGK simulation for N/MEMS Hydrodynamic. The qualitative and quantitative agreement between our LBGK simulations and experimental data over a wide range of pressure $0.1 \leq p \leq 1000$ Torr and frequency variation $0.001 \leq \tau \omega \leq 10$ constitutes a remarkable achievement for the kinetic methodology applied in this work. The precise determination of fluid forces and, thus, device quality factors for diverse geometrical configurations in widely different operation regimes is of fundamental importance in 


$$
\begin{array}{ccc}
\mathbf{v}_{i} / c_{-}^{a} & \text { states } & w_{i} \\
(0,0) & 1 & 0.233150669132352000 \\
(1,0) & 4 & 0.107306091542219000 \\
( \pm 1, \pm 1) & 4 & 0.057667859888794800 \\
(2,0) & 4 & 0.014208216158450700 \\
( \pm 2, \pm 2) & 4 & 0.001011937592673570 \\
(3,0) & 4 & 0.000245301027757717 \\
( \pm 1, \pm 2) & 8 & 0.005353049000513770 \\
( \pm 1, \pm 3) & 8 & 0.000283414252994198
\end{array}
$$

\footnotetext{
${ }^{a}$ Lattice Speed: $c=1.19697977039307 \sqrt{\theta}$
}

Table 2: Model Parameters D2Q37

advancing the design of future N/MEMS devices.

\section{Acknowledgments}

The authors acknowledge Dr. Hudong Chen and Xiaowen Shan from EXA corporation for their support in the development of the employed numerical tools. This work was funded by the National Science Foundation (NSF) under Grant No. CBET-0755927.

\section{A Appendix: The LBGK model D2Q37}

Velocity abscissae and weights of the D2Q37 lattice model [23, 22] is presented in table 2

\section{References}

[1] R. B. Bhiladvala and Z. J. Wang. Effect of fluids on the Q factor and resonance frequency of oscillating micrometer and nanometer scale beams. Phys. Rev. E, 69:036307, 2003.

[2] G. Binnig, C. F. Quate, and C. Gerber. Atomic force microscope. Phys. Rev. Lett., 56:930, 1982.

[3] R. B. Bird, W. E. Stewart, and E. N. Lightfoot. Transport Phenomena. Wiley \& Sons, 2002.

[4] C. Cercignani. Mathematical methods in kinetic theory. Plenum Pub. Corp., 1969.

[5] S. Chapman and T. G. Cowling. The Mathematical Theory of Non-Uniform Gases. Cambridge, 1970. 
[6] H. Chen, S. A. Orszag, and I. Staroselsky. Macroscopic description of arbitrary Knudsen number flow using Boltzmann-BGK kinetic theory. J. Fluid Mech., 574:495-505, 2007.

[7] A. N. Cleland and M. L. Roukes. Ananometre-scale mechanical electrometer. Nature, 392:161, 1998.

[8] C. E. Colosqui, H. Chen, X. Shan, I. Staroselsky, and V. Yakhot. Propagating high-frequency shear waves in simple fluids. Phys. Fluids, 21:013105, 2009.

[9] C. E. Colosqui and V. Yakhot. Lattice Boltzmann Simulation of a Non-Newtonian Oscillating Flow in a High-Frequency Limit. Int. J. Mod. Phys. C, 18(04):473-482, 2007.

[10] K. L. Ekinci, X. M. Huang, and M. L. Roukes. Ultrasensitive nanoelectromechanical mass detection. Appl. Phys. Lett., 84:4469, 2004.

[11] K. L. Ekinci, D. M. Karabacak, and V. Yakhot. Universality in Oscillating Flows. Phys. Rev. Lett., 101:26, 2008.

[12] D. Evans and G. Morriss. Statistical mechanics of nonequilibrium liquids. Cambridge, 2008.

[13] H. Grad. On the kinetic theory of rarefied gases. Commun. Pure Appl. Math., 2(4), 1949.

[14] N. G. Hadjiconstantinou. Oscillatory shear-driven gas flows in the transition and free-molecular-flow regimes. Phys. Fluids, 17:100611, 2005.

[15] D. M. Karabacak, V. Yakhot, and K. L. Ekinci. High-frequency nanofluidics: An experimental study using nanomechanical resonators. Phys. Rev. Lett., 98:254505, 2007.

[16] T. Kouh, D. Karabacak, D. H. Kim, and K. L. Ekinci. Diffraction effects in optical interferometric displacement detection in nanoelectromechanical systems. Appl. Phys. Lett., 86:013106, 2005.

[17] L. D. Landau and E. M. Lifshitz. Fluid mechanics. Pergamon, 1959.

[18] E. Lauga, M. P. Brenner, and H. A. Stone. Microfluidics: the no-slip boundary condition. Handbook of Experimental Fluid Dynamics, 2005.

[19] J. H. Park, P. Bahukudumbi, and A. Beskok. Rarefaction effects on shear driven oscillatory gas flows: A direct simulation Monte Carlo study in the entire Knudsen regime. Phys. Fluids, 16:317, 2004.

[20] M. R. Paul and M. C. Cross. Stochastic dynamics of nanoscale mechanical oscillators immersed in a viscous fluid. Phys. Rev. Lett., 92(23):235501-235501, 2004.

[21] J. E. Sader. Frequency response of cantilever beams immersed in viscous fluids with applications to the atomic force microscope. J. Appl. Phys., 84:64, 1998. 
[22] X. Shan and H. Chen. a General Multiple-Relaxation Boltzmann Collision Model. Int. J. Mod. Phys. C, 18(04):635-643, 2007.

[23] X. Shan, X. F. Yuan, and H. Chen. Kinetic theory representation of hydrodynamics: a way beyond the Navier-Stokes equation. J. Fluid Mech., 550:413-441, 2006.

[24] E. O. Tuck. Calculation of unsteady flows due to small motions of cylinders in a viscous fluid. J. Eng. Math., 3(1):29-44, 1969.

[25] H. C. Weng. A challenge in Navier-Stokes-based continuum modeling: Maxwell-Burnett slip law. Phys. Fluids, 20:106101, 2008.

[26] V. Yakhot and C. E. Colosqui. Stokes'second flow problem in a high-frequency limit: application to nanomechanical resonators. J. Fluid Mech., 586:249-258, 2007.

[27] R. Zhang, X. Shan, and H. Chen. Efficient kinetic method for fluid simulation beyond the Navier-Stokes equation. Phys. Rev. E, 74(4), 2006. 